\title{
Descripción Anatómica e Histológica del Aparato Digestivo de la Sabaleta (Brycon henni), Antioquia, Colombia
}

\author{
Anatomical and Histological Description of the Digestive Tract of the Sabaleta \\ (Brycon henni), Antioquia, Colombia
}

Luis F. Londoño-Franco ${ }^{1,4}$, Laura M. Laverde-Trujillo², Fabián G. Muñoz-García ${ }^{3}$

\section{Resumen}

El presente estudio hace un análisis del patrón anatómico e histológico del sistema digestivo de la sabaleta (Brycon henni). Fueron capturados seis individuos machos y hembras adultos en la quebrada Guaracú, Antioquia, Colombia, entre mayo y junio de 2016. Los peces pesaron $151.2 \mathrm{~g}$ y midieron $22.7 \mathrm{~cm}$ de longitud total. Los órganos del aparato digestivo fueron fijados en formol buferado al $10 \%$ y procesados con técnicas histológicas de rutina. Las estructuras más resaltantes, debido a su patrón anatómico específico y complejidad histológica fuero las branquiespinas (4), los ciegos pilóricos (13 en hembras y 8 en machos), el hepato-páncreas voluminoso y lobulado, y el intestino anterior, medio y posterior con vellosidades y microvellosidades. La sabaleta, según el patrón digestivo en cuanto a la clase de dientes, branquiespinas, estómago, longitud y estructura intestinal, sugiere que es una especie de hábito alimenticio omnívoro, pero con tendencia carnívora.

Palabras clave: peces nativos; morfología; caracterización

\section{AbSTRACT}

The present study describes the anatomical and histological pattern of the digestive system of the 'sabaleta' (Brycon henni). Six adult male and female individuals were fished in Guaracu creek, Antioquia, Colombia, between May and June 2016. The fish weighed $151.2 \mathrm{~g}$ and were $22.7 \mathrm{~cm}$ in total length. The organs of the digestive system were fixed in

${ }^{1}$ Facultad de Ciencias Agrarias, Politécnico Colombiano Jaime Isaza Cadavid, Medellín, Colombia

${ }^{2}$ Laboratorio Histiopath, Medellín, Colombia

${ }^{3}$ Facultad de Medicina Veterinaria, Universidad Antonio Nariño, Popayán, Colombia

${ }^{4}$ Email: lflondono@elpoli.edu.co

Recibido: 26 de octubre de 2016

Aceptado para publicación: 20 de mayo de 2017 
$10 \%$ buffered formalin and processed with routine histological techniques. The most remarkable structures, due to their specific anatomical pattern and histological complexity, were the gill rakers (4), the pyloric caeca (13 in females and 8 in males), the bulky and lobate hepato-pancreas, and the anterior, middle and posterior intestine with villi and microvilli. The 'sabaleta', according to the digestive pattern as to teeth, gill rakers, stomach, and intestinal structure and length, suggests that it is a kind of omnivorous food habit species, but with a carnivorous tendency.

Key words: native fish; morphology; characterization

\section{INTRODUCCIÓN}

La sabaleta (Brycon henni), pez de agua dulce endémica de la región andina. Pertenece al orden Characiformes, familia Characidae, subfamilia Bryconidae, conformada por cerca de 40 familias (Pineda et al., 2007). Es una de las especies migratorias más importantes de los pequeños ríos que se originan en la cordillera Central de Colombia.

Esta especie se distribuye en la región central de Colombia, en el Alto y Bajo río Cauca, así como en el río Magdalena, en la región pacífica en los ríos San Jorge, San Juan y en el Patía (Dahl, 1971; MaldonadoOcampo et al., 2005), y es muy apreciada por los habitantes de las zonas cafeteras del país. Se encuentra en peligro de extinción por la sobrepesca y las deficientes condiciones de su hábitat natural, debido a la construcción de represas que obstruyen los canales, la alta contaminación por minería y productos químicos agropecuarios; además de los asentamientos de personas y animales cerca de ríos y quebradas donde habita.

El presente estudio hace un análisis del patrón anatómico e histológico del sistema digestivo, con el objeto de ampliar el conocimiento existente que permita mejorar la nutrición y alimentación de la sabaleta.

\section{Materiales y Métodos}

Se trabajó con seis individuos machos y hembras adul tos de sabal eta (Brycon henni) capturados del medio natural, en la quebrada Guaracú, del municipio de San Jerónimo, Antioquia, Colombia, entre mayo y junio de 2016. Los peces tuvieron un peso promedio de $151.2 \mathrm{~g}$ y presentaron una longitud total promedio de $22.7 \mathrm{~cm}$ (Figura 1).

Los peces fueron anestesiados con una sobredosis de quenaldine $(0.2 \mathrm{~g} / 1)$ y sacrificados por sección medular, al nivel de la espina supraoccipital, teniendo en cuenta las guías para el manejo ético de peces para la investigación (Branson, 2008; AVMA, 2011). La necropsia se realizó mediante disecciones ventral y longitudinal al cuerpo para exponer la cavidad celómica y proceder a identificar y describir la disposición, apariencia y relaciones del esófago, estómago, intestino, ciegos pilóricos, hígado y páncreas.

Se evaluaron los índices de factor de condición $\mathrm{K}$ (peso total vs longitud total), $\mathrm{K}=100\left(\mathrm{~W} / \mathrm{L}^{3}\right)$ (Ricker, 1975); hepatosomático (peso del hígado vs peso total), gonadosomático (peso del ovario o testículo vs peso total); así como el coeficiente intestinal $(\mathrm{CI})$ donde $\mathrm{CI}=\mathrm{L} / \mathrm{L}_{\mathrm{s}} \mathrm{y} \mathrm{L}_{\mathrm{i}}$ es la longitud intestinal y $\mathrm{L}_{\mathrm{s},}$ es longitud total (Albrecht $e t$ 




Figura 1. Ejemplares adultos de sabaleta (Brycon henni). Macho (arriba) y hembra (abajo)

al., 2001; Treer et al., 2008). Para la medición se utilizó un ictiómetro Krauss \& Henke KH-PISCIS-50-22, y para el pesaje se empleó una balanza digital Lexus para $20 \mathrm{~kg}$ y de $1 \mathrm{~g}$ de precisión. Asimismo, el $\mathrm{pH}$ del esófago, estómago e intestino fue determinado mediante la tirilla reactiva universal $\mathrm{pH}$ de Merck ${ }^{\circledR}$.

Los órganos fueron extraídos y fijados en formol al 10\%. El análisis histológico se hizo en un laboratorio comercial (Histopath, Medellín). Las estructuras se sometieron a un proceso de deshidratación en concentraciones crecientes de etanol y se incluyeron en parafina (Agarwal, 1996). Se realizaron cortes transversales semiseriados de $5 \mu \mathrm{m}$ de espesor, en un micrótomo rotatorio (Leica RM2125 RTS) y los cortes se tiñeron con hematoxilina-eosina (HE). Para el estudio histológico se tuvo en consideración la presencia y componentes de las capas del tubo digestivo, así como las glándulas anexas del aparato digestivo (hígado y páncreas), descritas según Ferguson (2007) y Genten et al. (2009).

\section{Resultados y Discusión}

En el Cuadro 1 se observan los datos de peso ( $\mathrm{g}$ ) de los seis peces y de sus órganos, así como la longitud $(\mathrm{cm})$ total e intestinal. En el Cuadro 2 se presentan los resultados del factor de condición (k) e índices hepatosomáticos y gonadosomáticos, así como del coeficiente intestinal. Estos valores indican que los ejemplares estudiados están suficientemente maduros, desarrollados, en buenas condiciones de salud y su adaptación es adecuada a la ecología y factores ambientales prevalecientes (Wilson y Castro, 2011). La gran diferencia en el peso de las gónadas entre sexos a favor de las hembras, así como la mayor longitud total de las hembras demuestra que estas son más precoces en su desarrollo (Lenis et al., 2015).

La longitud del intestino fue de $16 \pm 2$ $\mathrm{cm}$, y presentó una relación entre la longitud intestinal $v s$ longitud total de 0.7 ; lo cual es propio de peces con hábito omnívoro (De Silva y Anderson, 1995). Sin embargo, la 
Cuadro 1. Peso (g) de seis peces Brycon henni y de órganos y longitud (cm) total e intestinal, según sexo, capturados en Antioquia, Colombia (2016)

\begin{tabular}{cccccc}
\hline Sexo & Peso total & $\begin{array}{c}\text { Peso del } \\
\text { hígado }\end{array}$ & $\begin{array}{c}\text { Peso de la } \\
\text { gónada }\end{array}$ & $\begin{array}{c}\text { Longitud } \\
\text { total }\end{array}$ & $\begin{array}{c}\text { Longitud } \\
\text { intestinal }\end{array}$ \\
\hline Hembra & 152.9 & 29.6 & 32.8 & 24.2 & 17.0 \\
Macho & 149.4 & 19.1 & 14.2 & 21.2 & 14.0 \\
\hline
\end{tabular}

Cuadro 2. Índices hepato-somático, gónado-somático y coeficiente intestinal en sabaletas (Brycon henni), según sexo (Antioquia, Colombia, 2016)

\begin{tabular}{ccccc}
\hline Índice & $\begin{array}{c}\text { Factor de } \\
\text { condición }(\mathrm{K})\end{array}$ & $\begin{array}{c}\text { Hepato- } \\
\text { somático }\end{array}$ & $\begin{array}{c}\text { Gonado- } \\
\text { somático }\end{array}$ & $\begin{array}{c}\text { Coeficiente } \\
\text { intestinal }\end{array}$ \\
\hline Hembras & 1.09 & 0.193 & 0.214 & 0.702 \\
Machos & 1.57 & 0.127 & 0.095 & 0.661 \\
\hline
\end{tabular}

sabaleta tiene tendencia carnívora pues presenta dientes (Botero-Botero y Ramírez-Castro, 2011). Asimismo, el coeficiente intestinal menor de 1 (0.661-0.702) en ambos sexos sugiere intestinos de especies con hábito alimenticio carnívoro (Karachle y Stergiou, 2010).

El pH en el esófago, estómago y en intestino anterior medio y posterior fue de 6.8 , $4.2,7.1,7.5$ y 7.5 , respectivamente.

En la Figura 2 se aprecia la presencia de una lengua voluminosa y bien diferenciada, ocupando gran parte de la cavidad bucal y los dientes a nivel de los labios (viliformes), más desarrollados en la mandíbula inferior. Según la ubicación y forma de los dientes se puede deducir que la sabaleta puede tragar presas enteras y que los dientes les permiten separar en trozos e incluso raspar el fondo en busca de alimento, pudiendo, además, in- gerir los detritos presentes (Vásquez-Torres, 2001; Gómez et al., 2010). Esto coincide con los estudios de contenido estomacal reportados por Botero-Botero y Ramírez-Castro (2011), quienes estudiaron la ecología trófica de la sabaleta, encontrando material vegetal, insectos, crustáceos y moluscos; es decir, un hábito alimenticio omnívoro.

Hacia el interior de la lengua se encuentra un tejido conectivo denso y gran cantidad de adipocitos, además de plexos nerviosos y vasos sanguíneos. Asimismo, se pueden apreciar algunas papilas gustativas fungiformes (Fig. 3A), lo que permite inferir que esta especie podría captar algunos sabores de los alimentos, lo cual determina, posiblemente, una selectividad de alimentos o presas. La mucosa oral presenta un epitelio escamoso estratificado con presencia de algunas células caliciformes, además de una capa de músculo esquelético longitudinal (Figura 3B). 

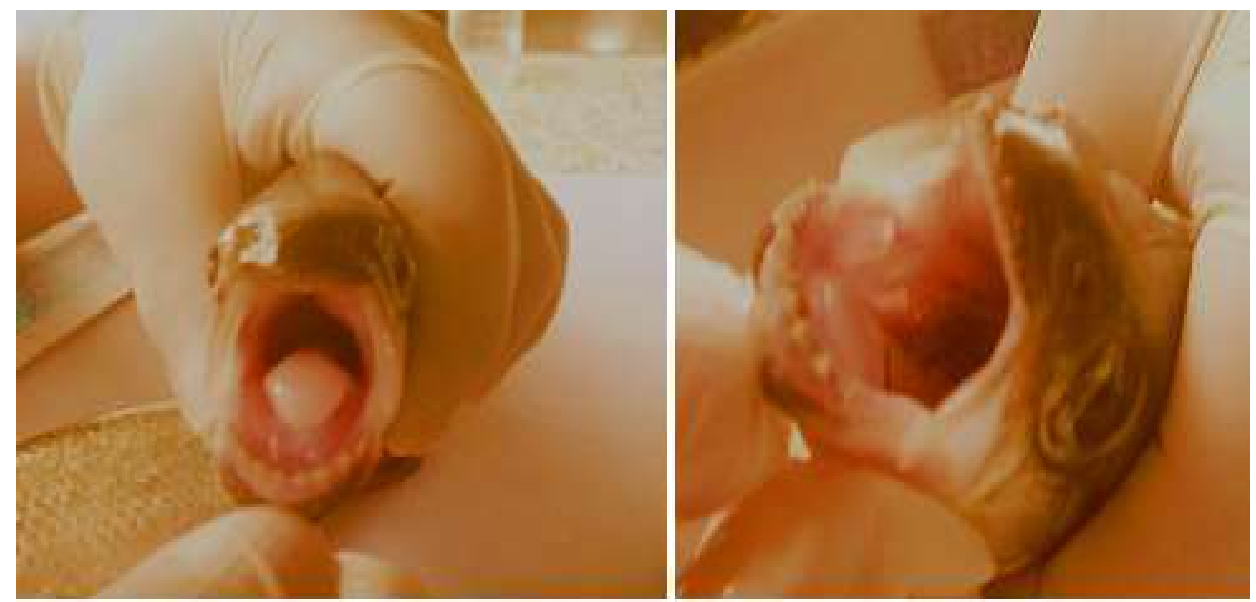

Figura 2. Boca mostrando la lengua y dientes de la sabaleta (Brycon henni)
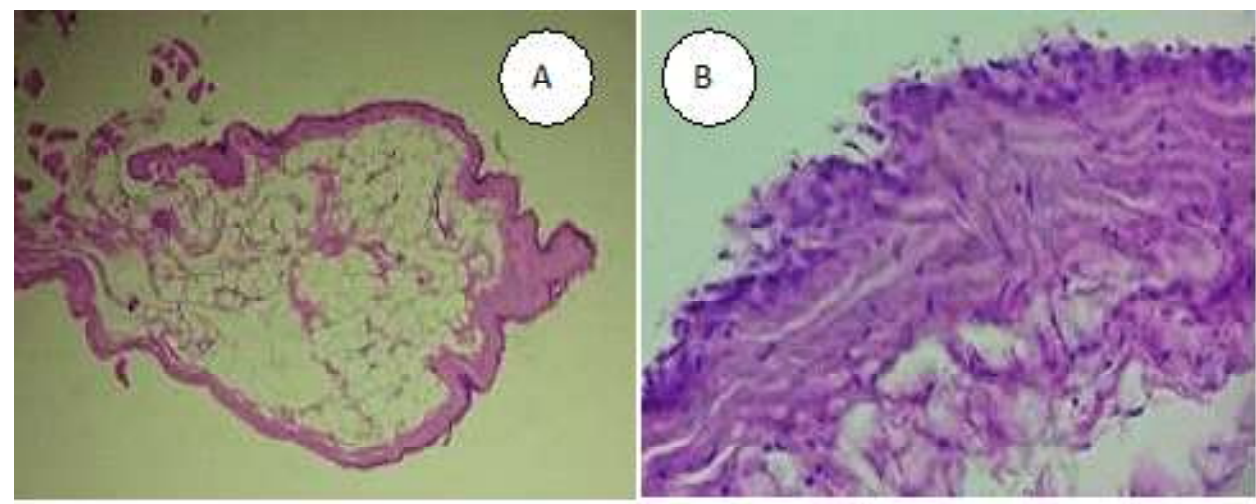

Figura 3. Corte histológico de lengua (A) y mucosa oral de sabaleta (B) (Brycon henni) (HE, 20X)

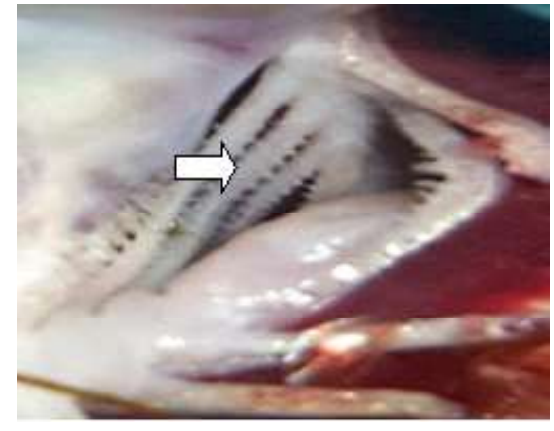

Figura 4. Cuatro branquiespinas (flecha) en la parte caudal de la cavidad bucal de la sabaleta (Brycon henni)
La Figura 4 muestra las cuatro branquiespinas cortas, bien definidas y separadas entre sí, lo cual es característico de especies semicarnívoras y algunas omnívoras (Vásquez-Torres, 2004). Esta estructura les permite a los peces una mayor filtración de los alimentos para su aprovechamiento, especialmente partículas y sólidos disueltos en el agua (Torres-Salinas, 2011). El número de branquiespinas y el espacio entre las mismas puede indicar una baja capacidad filtradora de la sabaleta con respecto a otras especies. Por ejemplo, en algunas especies como la cachama negra (Colossoma macropomum) 

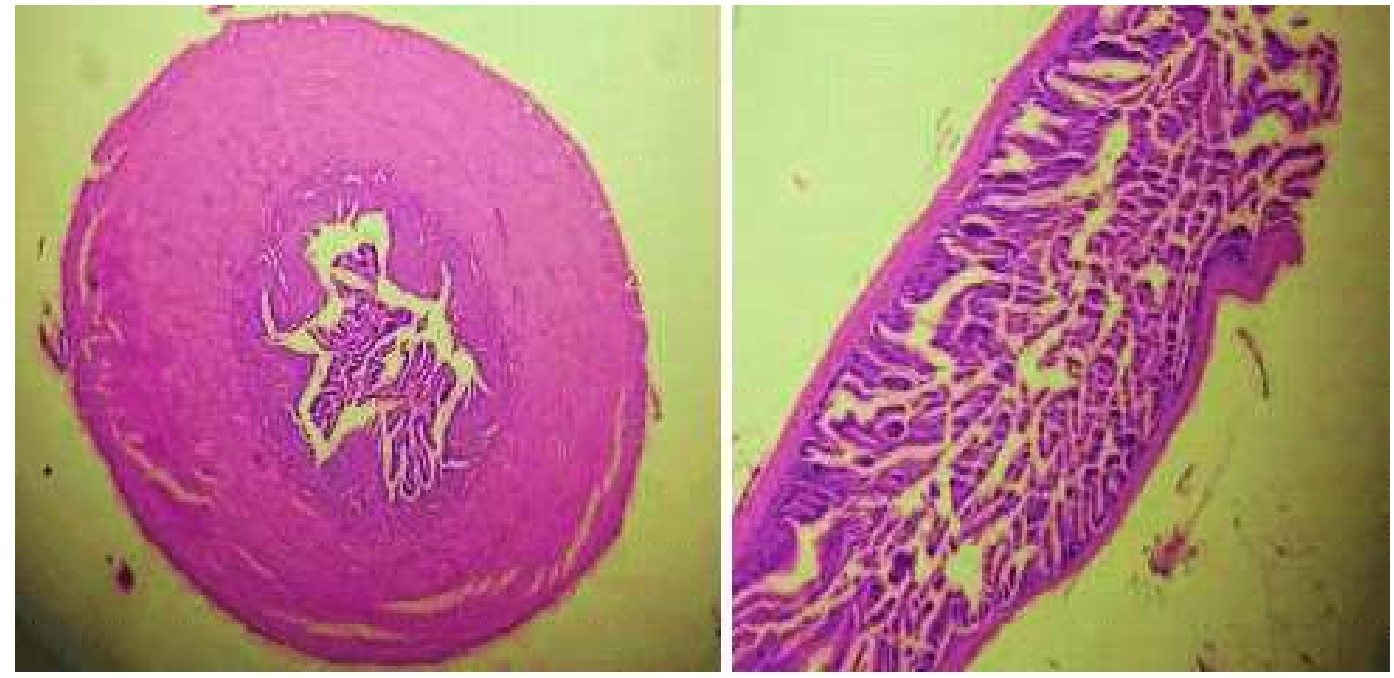

Figura 5. Cortes histológicos de esófago de sabaleta (Brycon henni). Corte trasversal (izquierda) y longitudinal (derecha). (HE, 20X)

y el bocachico (Prochilodus magdalenae) se pueden encontrar más de 80 , resaltando su importancia en especies filtradoras (Woynarovich, 1988; Eslava, 2001).

La forma y disposición del tracto digestivo guarda similitud con aquellas de peces más desarrollados y evolucionados como los teleósteos (Ferguson, 2007; Roberts, 2012). Por otro lado, los órganos más desarrollados en cuanto a volumen y peso fueron las gónadas femeninas, seguido del hígado (Cuadro 1).

A nivel macroscópico, el esófago representa una pequeña dilatación del tubo digestivo. Está constituido por capas concéntricas de mucosa, submucosa, muscular y serosa, análogas del esófago de mamíferos y de la mayoría de peces teleósteos (Gómez et al., 2010). El esófago presenta pliegues mucosos longitudinales ramificados, compuestos por el borde de la mucosa y la submucosa que se interioriza. La mucosa está formada por un epitelio estratificado cilíndrico y la submucosa por tejido conectivo laxo, con presencia de fibras nerviosas, vasos sanguíneos y, en algunos casos, paquetes musculares longitu- dinales. La capa muscular es la más voluminosa y desarrollada, y está compuesta por músculo liso con una capa ancha, dispuesta de forma transversal, y otra más delgada, de forma longitudinal (Figura 5). Por último, la serosa es una mono-capa muy delgada de tejido simple plano. Los resultados difieren de Eslava (2000), quien en peces como el yamú (Brycon siebenthalae) y la cachama blanca (Piaractus brachyopomus) encontró músculo esquelético en vez de músculo liso en el esófago, lo que les permitiría devolver el alimento para remasticar o eliminar el alimento (Bakke et al., 2010).

El estómago típico de muchos peces suele tener forma sigmoidea (más pronunciado en carnívoros), con un saco ciego, más o menos alargado y dirigido caudalmente. El interior se suele dividir en tres regiones (cardias, fundus y región pilórica) (Wilson y Castro, 2011). En algunos peces solo se aprecian las regiones fúndica y pilórica (VásquezTorres, 2004). La mucosa contiene numerosos pliegues gástricos y es rica en glándulas secretoras de mucus, pepsina y ácido clorhídrico (Halver, 1989; Hepher, 1993; Mendoza et al., 2013). 


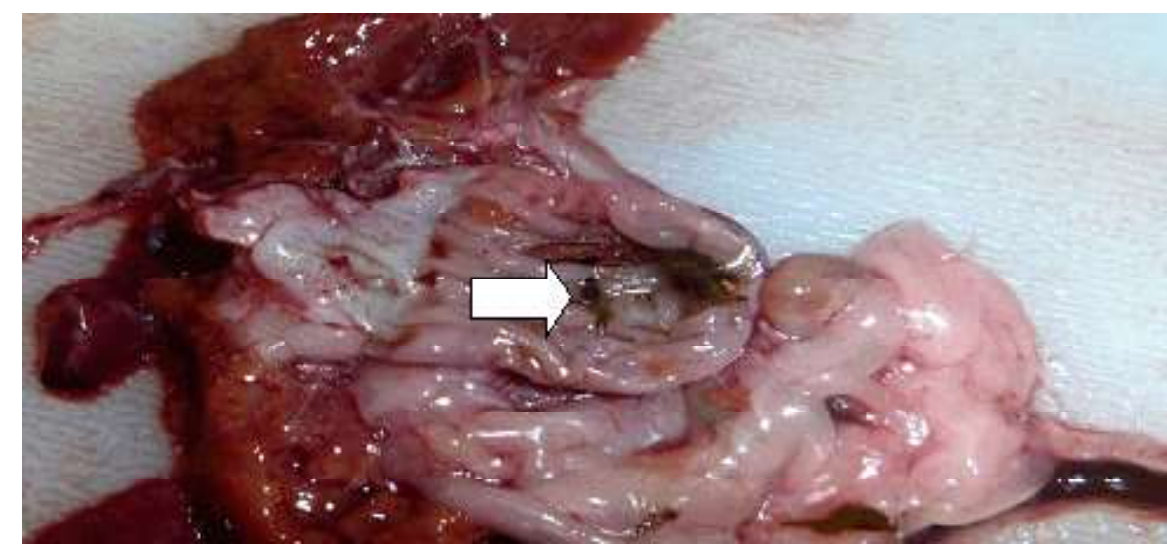

Figura 6. Estómago de sabaleta (Brycon henni) mostrando la capa interna de la mucosa (flecha). Se observa los pliegues gástricos
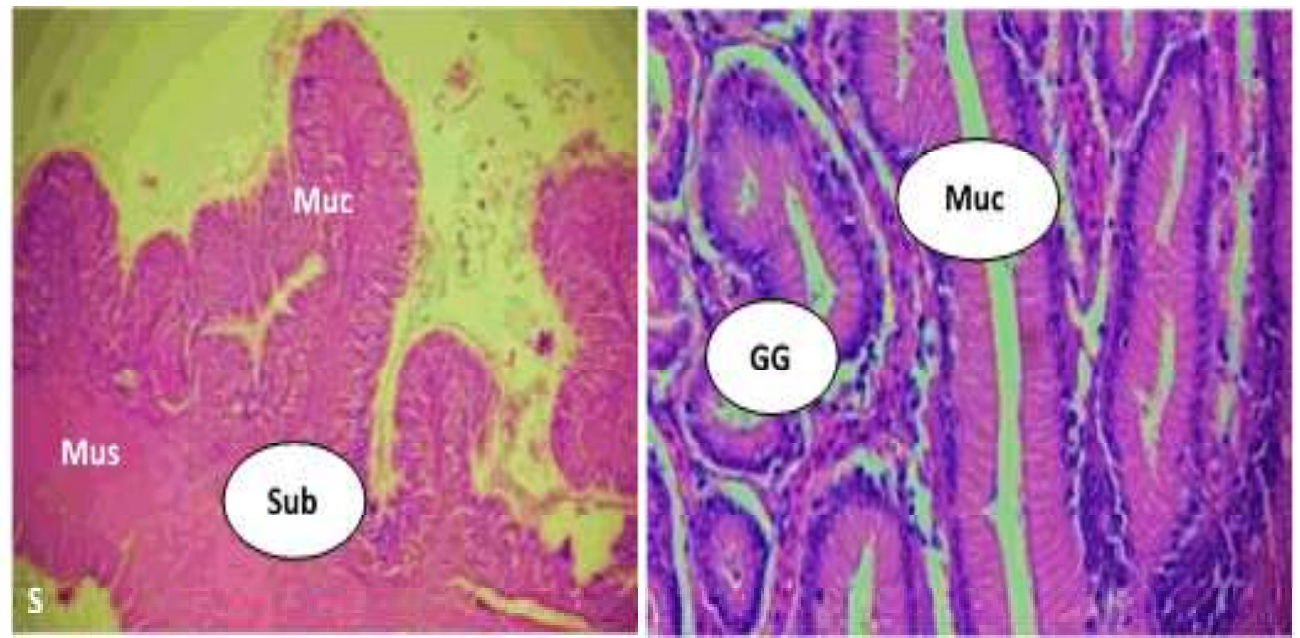

Figura 7. Histología del estómago de sabaleta (Brycon henni). En la foto izquierda se observa la capa serosa (S), muscular (Mus), submucosa (Sub) y mucosa (Muc). La foto derecha muestra la capa mucosa (Muc) con epitelio cilíndrico y numerosas glándulas gástricas (GG) (HE, 40X)

En la Figura 6 se distingue dos regiones a nivel de mucosa en el estómago; una de color blanquecino o claro hacia craneal que se correspondería con la región fúndica y otra de color más rosado u oscuro y de mayor tamaño que equivale a la región pilórica, con numerosos pliegues gástricos, coincidiendo con otros investigadores (Eslava, 2000; Vásquez-Torres, 2004).

En la Figura 7 se aprecia las características histológicas del estómago de la sabaleta. La mucosa está compuesta por un epitelio cilíndrico simple, seguido de una capa de 

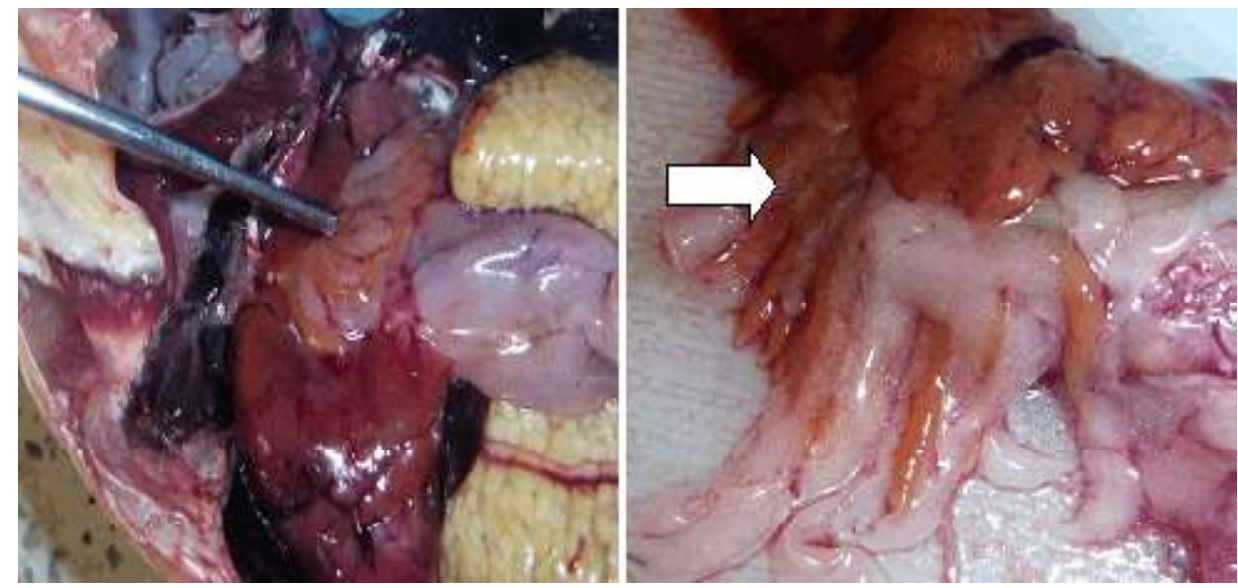

Figura 8. Ciegos pilóricos de sabaleta (Brycon henni), señalados con la pinza y la flecha

numerosas glándulas gástricas tubulares, en las cuales se observan gránulos de secreción altamente basófilos. La capa muscular de la mucosa es delgada. La submucosa presenta tejido conectivo laxo. La capa muscular presenta músculo liso con dos orientaciones: una transversal interna y una longitudinal externa, siendo de mayor espesor la capa transversal. La capa serosa está compuesta por epitelio simple plano, disposición común de peces teleósteos (Ferguson, 2007; Roberts, 2012).

Se encontraron 13 ciegos pilóricos en promedio en las hembras y ocho en los machos (Figura 8). Los ciegos pilóricos se relacionan íntimamente con el intestino anterior, el hígado y el estómago. Presentan una estructura anatómica similar al intestino, y cumplen funciones similares a los ciegos pilóricos de especies como la trucha arcoíris (Oncorhynchus mykiss), lubina rayada (Morone saxatilis), esturión blanco (Acipenser transmontanus), lubina marina (Centropristes striatus), reedfish (Polypterus senegalus) y robin marino (Prionotus carolinus) (Buddington y Diamond, 1987; Halver, 1989). El número de ciegos pilóricos puede ser muy variable, siendo hasta 70 en salmónidos, varios cientos en los túnidos y muy escasos (3-5) en espáridos, como la do- rada (Brycon moorei) y el sargo (Lagodon rhombioides) (Atensio et al., 2008). Asimismo, pueden estar ausentes en especies que carecen de estómago (Gil Cano et al., 2009). Además, se conoce que su número es mayor en especies carnívoras que herbívoras (Buddington y Diamond, 1987).

El número de ciegos pilóricos encontrado en este estudio coincide con el trabajo de Botero-Botero y Ramírez-Castro (2011) con sabaletas y el de Atensio et al. (2008) con rubio (Salminus affinis).

Los ciegos pilóricos poseen las cuatro capas concéntricas del tubo digestivo (mucosa, submucosa, muscular y serosa). La mucosa está formada por pliegues mucosos similares a los del intestino, largos y ramificados, compuestos por epitelio cilíndrico simple y escasas células caliciformes, y posee gran cantidad de gránulos basófilos; la submucosa presenta tejido conectivo laxo; la capa muscular está constituida por músculo liso, muy delgada y de forma transversal. La capa serosa está compuesta por epitelio plano simple (Figura 9).

En la Figura 10 se observa el hígado de color café, con fisuras que permiten la formación de lóbulos. El hígado lobulado difiere 

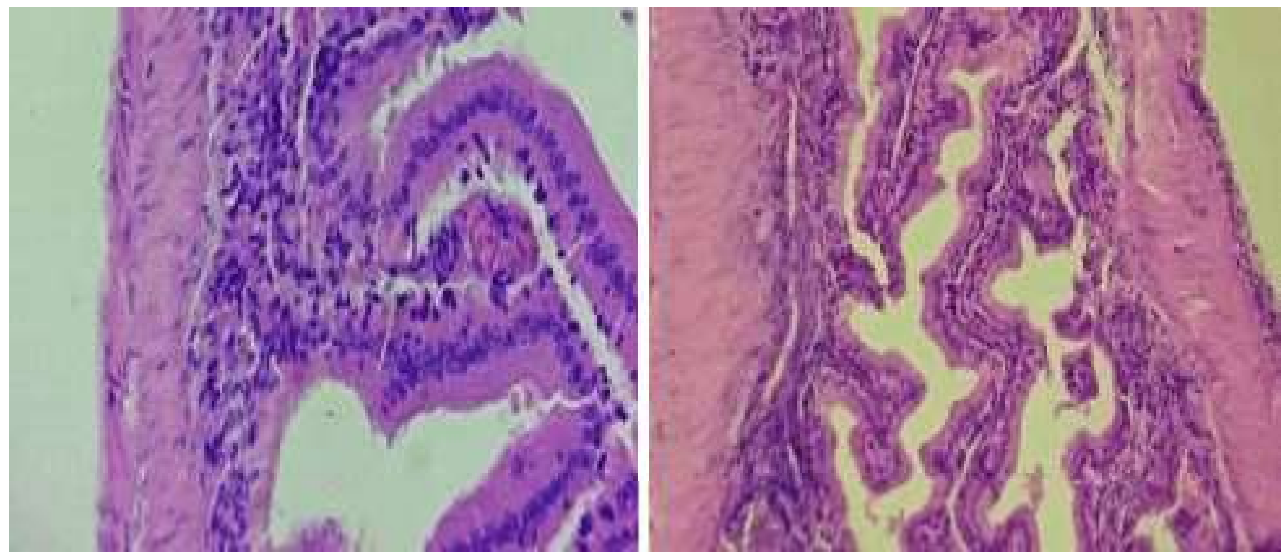

Figura 9. Cortes histológicos de los ciegos pilóricos de sabaleta (Brycon henni) (HE, 40X)

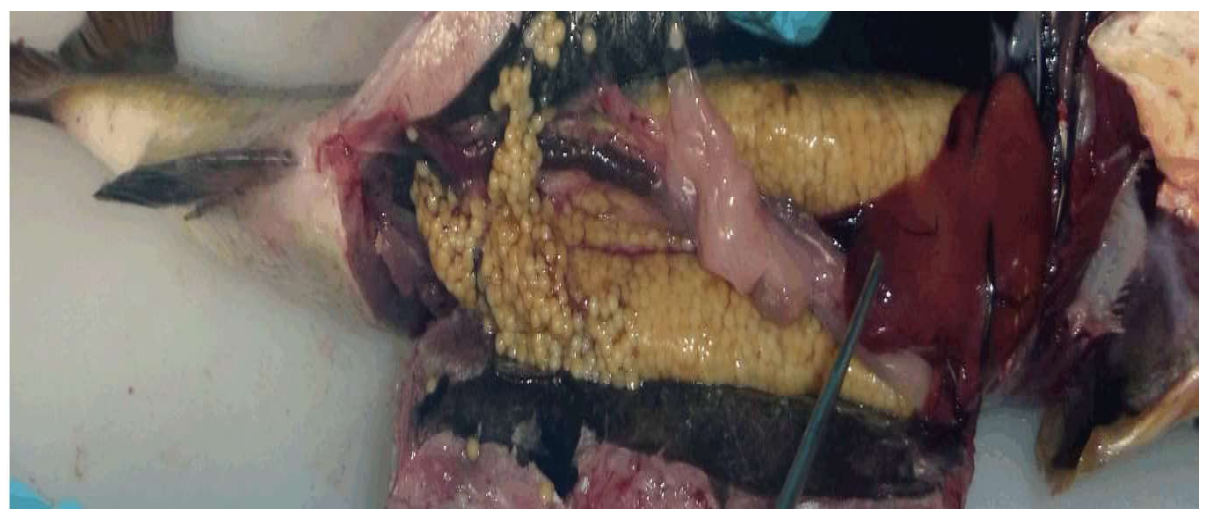

Figura 10. Hígado de sabaleta (Brycon henni). La pinza señala uno de los lóbulos principales o mayor

de otras especies como el nicuro (Pimelodus blochii) (López-Casas y Jiménez, 2007) y la cachama blanca (Vicentini et al., 2005; Mendoza et al., 2013). Por otro lado, es lobulado en la tilapia (Oreochromis niloticus) (Torres et al., 2010).

En el hepato-páncreas se observan hepatocitos, sinusoides hepáticos, canalículos biliares y tejido pancreático intrahepático (Figura 11). Los hepatocitos tienen forma poligonal o poliédrica y núcleo. Los sinusoides hepáticos se distribuyen de manera difusa entre los hepatocitos. En el interior del hígado se observa tejido pancreático, dispuesto de forma multifocal, rodeando algunos de los vasos sanguíneos (vena central). El tejido que conforma el páncreas en su gran mayoría es de tipo acinar, donde los acinos son de color basófilo y, la gran mayoría, con gránulos de secreción exocrina. Además, el hígado carece de la típica triada portal, propia de los mamíferos. Los conductos biliares y las ramas de la arteria hepática y vena porta se distribuyen por todo el parénquima, donde cordones de hepatocitos se disponen alrededor de los sinusoides. Suele haber tejido hematopoyético y abundantes macrófagos cargados de melanina (Gil Cano et al., 2009). 

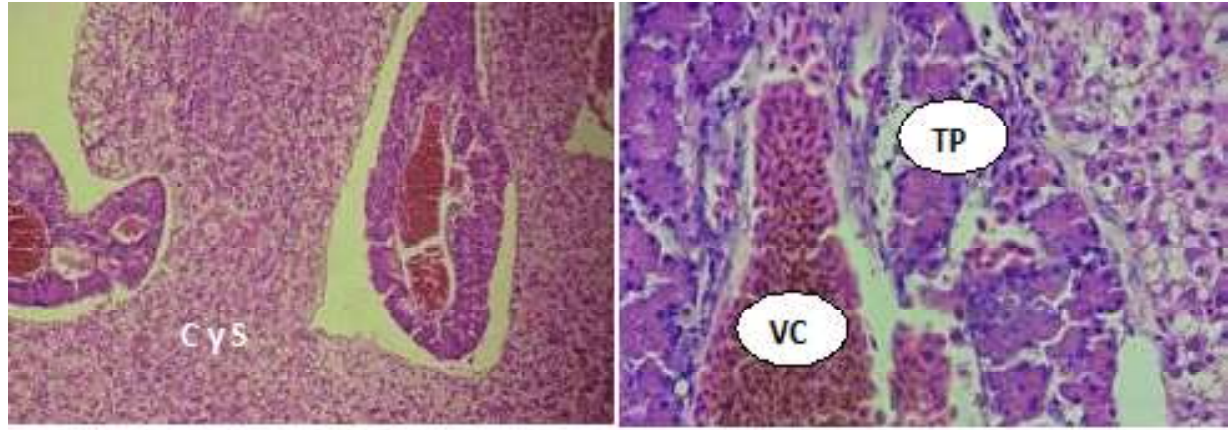

Figura 11. Histología del hígado (hepato-páncreas) de sabaleta (Brycon henni) con cordones (C) y sinusoides (S) hepáticos (foto izquierda). Hacia el interior del hígado se observa una gran vena (vena central - VC) con eritrocitos, seguido de tejido pancreático (TP) (foto derecha) (HE, 20X y 40X, respectivamente)

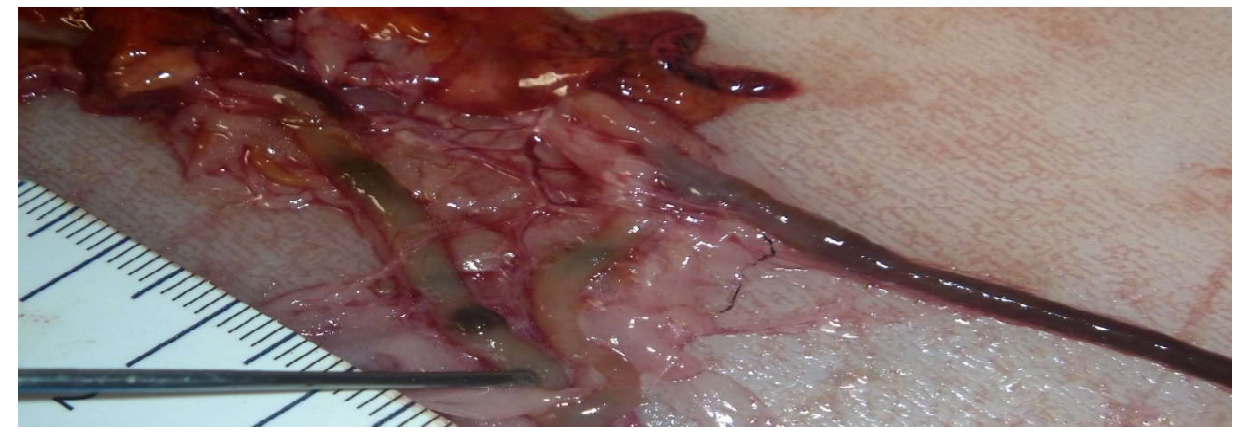

Figura 12. Vista del intestino de la sabaleta (Brycon henni). La pinza señala la ubicación de una de las asas intestinales

El páncreas presenta nódulos de disposición variable. En ciertas especies se localiza de forma difusa alrededor del intestino, entremezclado con la grasa mesentérica. En salmónidos, estos nódulos se disponen diseminados entre la grasa del mesenterio que fija los ciegos pilóricos (Lokka et al., 2013), mientras que en la carpa se dispersan en el hígado en torno a la vena porta, formando el hepatopáncreas (Wilson y Castro, 2011), y en otras especies se sitúan en el tejido subcapsular del bazo (Kuzir et al., 2012). El páncreas consta de una porción exocrina y otra endocrina (escasa o sin ella). Existe un conducto pancreático que normalmente se une al biliar antes de desembocar en el intestino anterior (Gil Cano et al., 2009).
En el intestino de sabaleta, a nivel macro, se diferencian dos asas intestinales (craneal y caudal), semejante a lo que ocurre en la cachama blanca (Mendoza et al., 2013). Esta especie se diferencia de los peces de hábito omnívoro y el nicuro que presentan cinco asas y el Brycon orbygnianus que presenta nueve asas intestinales (Seixas Filho et al., 2000). $\mathrm{Al}$ considerar que las asas intestinales permiten el transporte lento de la ingesta a fin de aumentar el tiempo de absorción de los nutrientes (García et al., 2008), se deduce que la sabaleta presenta un tránsito y una capacidad de absorción de nutrientes rápida (Figura 12). El intestino presenta un diámetro similar de aproximadamente $3 \mathrm{~mm}$ en toda su longitud. En la piarte final (intestino poste- 

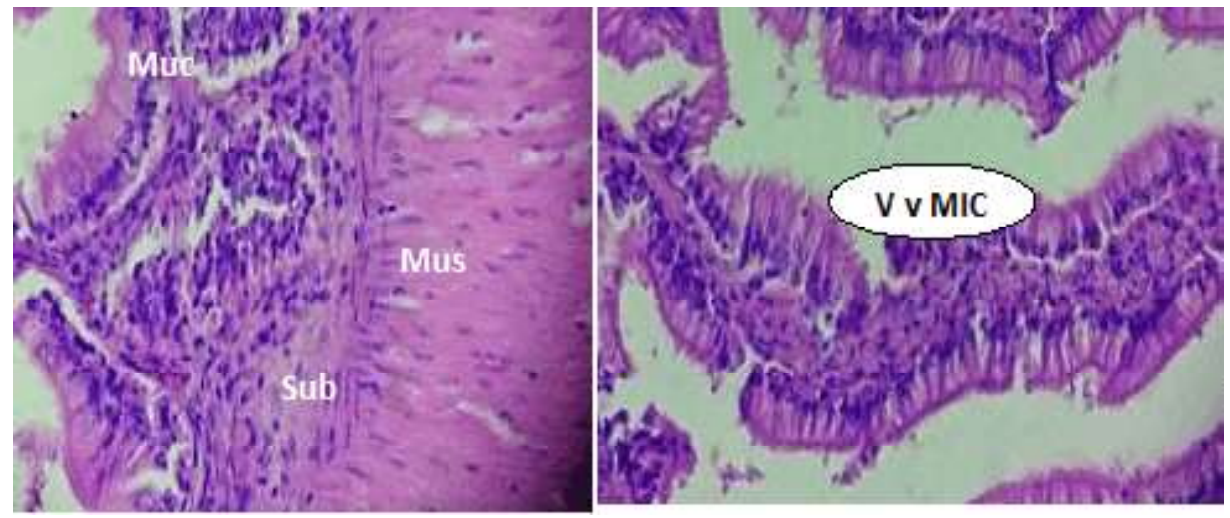

Figura 13. Intestino anterior de la sabaleta (Brycon henni). Foto izquierda: capa mucosa (Muc), submucosa (Sub) y muscular (Mus). Foto derecha: vellosidades y microvellosidades del epitelio intestinal (V y Mic) (HE, 40x)


Figura 14. Intestino medio de la sabaleta (Brycon henni). Se observan múltiples vellosidades y microvellosidades (V y Mic) y el epitelio (Ep) de enterocitos en borde de cepillo o chapa estriada (HE, 40X)

rior) se adelgaza para desembocar en la papila urogenital (Figura 15).

El intestino anterior está constituido por cuatro capas concéntricas: mucosa, submucosa, muscular y serosa (Figura 13). La mucosa está compuesta por un epitelio cilíndrico simple con vellosidades y microvellosidades en el borde apical y se visualizan pocas células caliciformes. La submucosa está formada por tejido conectivo laxo con abundantes capilares sanguíneos, vasos linfáticos, fibroblastos, fibras conectivas y ocasionalmente linfocitos aislados. La mus- cular de la mucosa es una capa de músculo liso muy delgada. La muscular está compuesta por dos capas: una transversal y una longitudinal; siendo de mayor espesor la capa transversal y entre ambas se observa un infiltrado celular monocítico (linfocitos) y vasos sanguíneos. La capa serosa está compuesta por un epitelio plano simple.

El intestino medio está igualmente conformado por las mismas capas concéntricas del intestino anterior. La mucosa está compuesta por finas vellosidades, las cuales presentan ramificaciones. El epitelio es simple 


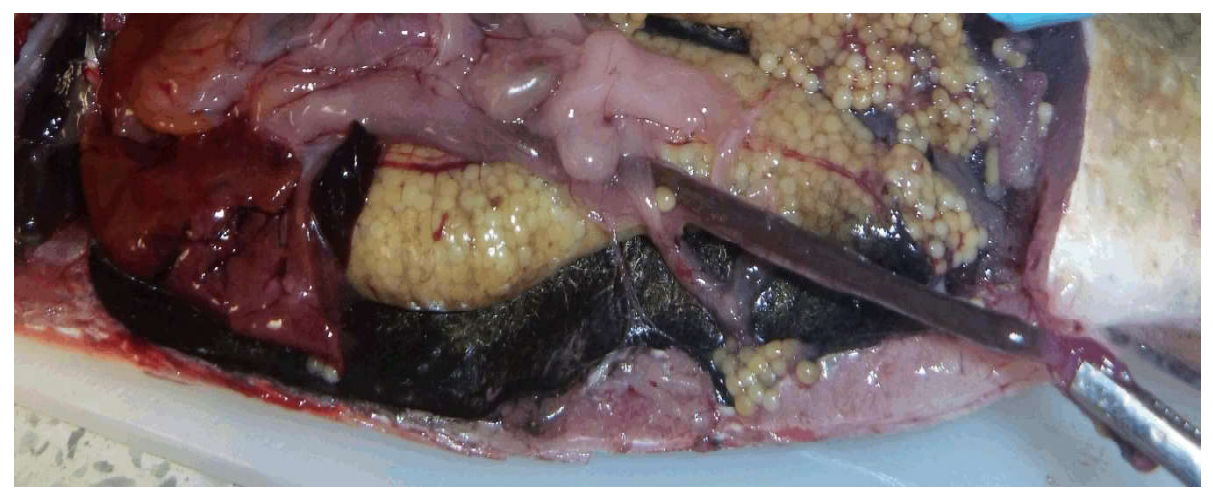

Figura 15. Intestino posterior de la sabaleta (Brycon henni). Se observa la desembocadura en la papila urogenital
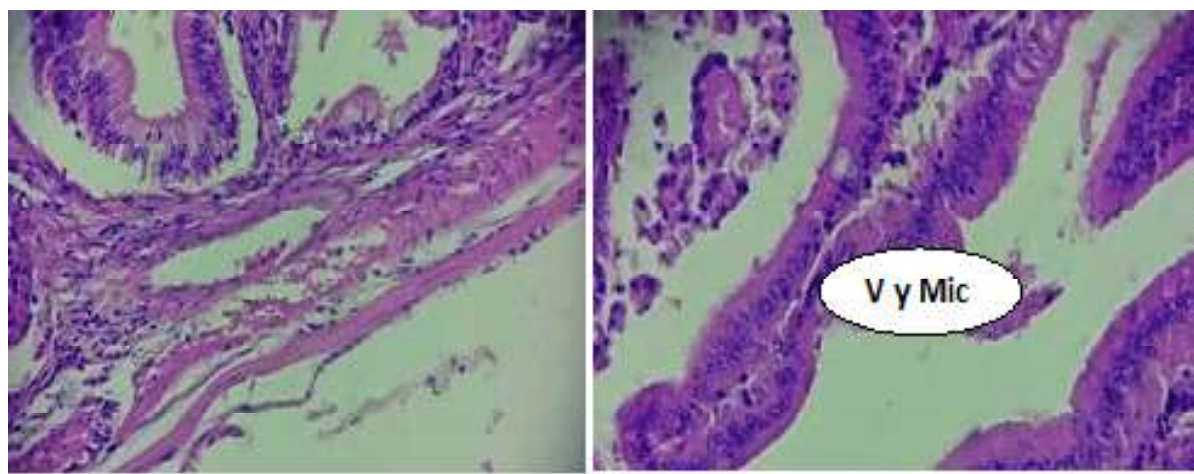

Figuras 16. Histología de intestino posterior, ilustrando las capas histológicas y con presencia de vellosidades y microvellosidades (V y Mic) (HE, 20X y $40 \mathrm{X}$, respectivamente)

cilíndrico con microvellosidades en el borde apical y se visualizan pocas células caliciformes (Figura 14). La submucosa está formada por tejido conectivo laxo con abundantes capilares sanguíneos y vasos linfáticos, fibroblastos, fibras conectivas y, ocasionalmente, linfocitos aislados. La capa muscular de la mucosa se encuentra ausente. La capa muscular propia está compuesta por dos capas, una transversal y una longitudinal, siendo de mayor espesor la capa transversal, con presencia de vasos sanguíneos entre ambas. La capa serosa está compuesta por un epitelio simple plano.
El intestino posterior contiene las capas concéntricas mencionadas en los otros dos segmentos intestinales (Figura 16). La mucosa está compuesta por un epitelio estratificado cilíndrico con la presencia de abundantes células caliciformes con núcleos basales y vacuolas de secreción; además, presenta vellosidades y microvellosidades en el borde apical. La submucosa está formada por tejido conectivo laxo con abundantes capilares sanguíneos y vasos linfáticos, fibroblastos, fibras conectivas y, ocasionalmente, linfocitos aislados. La capa muscular de la mucosa se encuentra ausente. La capa 
muscular propia está compuesta por dos capas delgadas: una transversal y la otra longitudinal, siendo de mayor espesor la capa transversal.

En resumen, la estructura macro y microscópica del intestino de la sabaleta es similar a la de la gran mayoría de peces teleósteos, especialmente a los de hábitos carnívoros (Seixa Filho et al., 2001).

\section{Conclusiones}

- Las estructuras que merecen mayor atención, debido a su patrón anatómico específico y complejidad histológica, en la sabaleta (Brycon henni) son: las branquiespinas (4), los ciegos pilóricos (13 en hembras y 8 en machos), el hepato-páncreas voluminoso y lobulado, $\mathrm{y}$ el intestino anterior, medio y posterior con vellosidades y microvellosidades.

- La sabaleta, según el patrón digestivo en cuanto a la clase de dientes, branquiespinas, estómago, longitud y estructura intestinal, sugiere que es una especie de hábito alimenticio omnívoro, pero con tendencia carnívora.

\section{Literatura Citada}

1. Agarwal NK. 1996. Fish reproduction. New Delhi, India: APH Pub. 157 p.

2. Albrecht MP, Ferreira MF, Caramaschi EP. 2001. Anatomical features and histology of the digestive tract of two related neotropical omnivorous fishes (Characiformes; Anostomidae). J Fish Biol 58: 419-430. doi: 10.1111/j.1095-8649.2001.tb02261.x

3. Atencio VJ, Hernández J, Pardo SC. 2008. Descripción morfológica del tubo digestivo de juveniles de rubio salminus affinis (Pisces: Characidae). Acta Biol Colomb 13: 99-112. .
4. [AVMA] American Veterinary Medical Association. 2011. AVMA guidelines on euthanasia. USA: AVMA. 86 p.

5. Bakke AM, Glover C, Krogdahl A. 2010. Feeding, digestion and absorption of nutrients. In: Grosell M, Farrell AP, Brauner CJ (eds). Fish physiology. The multifunctional gut of fish. San Diego, USA: Academic Press. p 57-110.

6. Botero Botero A, Ramirez Castro $\boldsymbol{H}$. 2011. Ecología trófica de la sabaleta Brycon henni (Pisces:Characidae) en el río Portugal de Piedras, Alto Cauca, Colombia. Rev MVZ Córdoba 16: 23492355.

7. Branson E. 2008. Fish welfare. New York: Blackwell. $316 \mathrm{p}$.

8. Buddington RK, Diamond J. 1987. Pyloric ceca of fish: a «new» absorptive organ. Am J Physiol 252: G65-G76.

9. Dahl G. 1971. Los peces del norte de Colombia. Bogotá: Inderena. 391 p.

10. De Silva SS, Anderson TA. 1995. Fish nutrition in aquaculture. London, UK: Chapman \& Hall. 320 p.

11. Eslava PR, Cruz PE, Vásquez W, Iregui C. 2000. Morfología del sistema digestivo de la cachama Piaractus brachypomus. Rev MVZ Unillanos 2(2): 22-31.

12. Eslava PR. 2001. Aspectos morfológicos del sistema digestivo del yamú (Brycon siebenthalae). Memorias VII Jornada de Acuicultura. Villavicencio, Colombia.

13. Ferguson H. 2007. Systemic pathology of fish: a text an atlas of normal tissues responses in teleosts, and their responses in disease. $2^{\text {nd }}$ ed. Iowa State University: Scotian Press. 289 p.

14. García RJ, Botero-Botero A, García Alzate C. 2008. Composición, estacionalidad y hábitat de los peces de la Quebrada Cristales, afluente del río La Vieja, Alto Cauca, Colombia. Rev Invest Univ Quindío 19: 115-121.

15. Genten F, Terwinghe E, Danguya A. 2009. Atlas of fish histology. USA: CRC Press. 224 p. 
16. Gil Cano F, Ayala Florenciano MD, López Albors O. 2009. Estómago, hígado, intestino, bazo y páncreas de los peces. España: Universidad de Murcia. 3 p.

17. Gómez E, Tovar O, Obando MJ, Hurtado H. 2010. Estudio histológico del tracto digestivo del pez Ariopsis seemanni (Ariidae). Rev Fac Cienc Básicas 6: 216-225.

18. Gouldin M, Carvalo M. 1982. Life history and management of the Tambaquí (Colossoma macropomun, caracidae), an important amazonian food fish. Rev Bras Zool 1(2): 107-133.

19. Halver J. 1989. Fish nutrition. $2^{\text {nd }}$ ed. San Diego, California: Academic Press. $798 \mathrm{p}$.

20. Hepher B. 1993. Nutrición de peces comerciales en estanque. México: Limusa. $406 \mathrm{p}$.

21. Karachle PK, Stergiou KI. 2010. Intestine morfhometrics of fishes: a compilation and analysis of bibliographic data. Acta Ichthiologic 40: 45-54.

22. Kuzir S, Gjurcevic E, Nejedli S, Bazdaric B, Kozaric $Z .2012$. Morphological and histochemical study of intestine in wild and reared European eel (Anguilla anguilla L). Fish Physiol Biochem 38: 625-633. doi: 10.1007/ s10695-011-9543-7

23. Lenis G, Cruz P, Ruales C. 2015. Reproducción inducida de la sabaleta Brycon henni: revisión bibliográfica. Rev Lasallista Invest 12: 211-220.

24. Lokka G, Austbo L, Falk K, Bjerkas I, Koppang EO. 2013. Intestinal morphology of the wild Atlantic salmon (Salmo salar). J Morphol 274: 859-876. doi: 10.1002/jmor.20142

25. López-Casas, Jiménez SF. 2007. Reproducción y hábitos alimenticios del nicuro, Pimelodus blochii (Valenciennes, 1840) (Pisces: Pimelodidae), en la ciénaga de Cachimbero, río Magdalena, Colombia. Actu Biol 29: 193-201.

26. Maldonado-Ocampo JA, OrtegaLara A, Usma-Oviedo JS. Villa-Navarro F, Vásquez, L, et al. 2005. Peces de los Andes de Colombia. Bogotá: Instituto Humboldt. 346 p.
27. Mendoza MA, Comas Corredor J, Romero C. 2013. Estudio histológico del sistema digestivo en diferentes estadios de desarrollo de la cachama blanca (Piaractus brachypomus). Rev Med Vet 25: 21-38.

28. Pineda H, Arboleda L, Echeverry A, Urcuqui S, Pareja D, Olivera $M$, Builes J. 2007. Caracterización de la diversidad genética en el pez Brycon henni (Characiformes: Characidae) en Colombia central por medio de marcadores RAPD. Rev Biol Trop 55: 1025 1035. doi: 10.15517/rbt.v55i3-4.5975

29. Ricker W. 1975. Computation and interpretation of biological statistics of fish populations. In: Canada: Bulletin Fisheries Research Board of Canada $191.382 \mathrm{p}$.

30. Roberts R. 2012. The aquatic environment. In: (Roberts RJ (ed). Fish pathology. $4^{\text {th }}$ ed. London: WileyBlackwell.p 11-39.

31. Seixas Filho JT, Bras JDM, Gomide ATM, Olivera MGA, Donzele JL, Menin E. 2001. Anatomia funcional e morfometría do intestino no Teleostei (Pisces) de água doce Surubim (Pseudoplatystoma coruscans Agassiz, 1829). Rev Bras Zootec 30: 1670-1680.

32. Seixas Filho JT, Bras JDM, Gomide ATM, Olivera MGA, Donzele JL, Menin E. 2000. Anatomia funcional e morfometría dos intestinos e dos cecos pilóricos do teleostei (Pisces) de água doce Brycon orbignyanus (Valenciennes, 1849). Rev Bras Zootec 29: 313-324.

33. Torres RGA, González PS, Peña SE. 2010. Anatomical, histological and ultraestructural description of the gills and liver in tilapia (Oreochromis niloticus) Int J Morphol 28: 703-712. doi: 10.4067/ S0717-95022010000300008

34. Torres-Salinas D. 2011. El sistema digestivo de peces teleósteos. Tesis de Magíster. México: Univ Nacional Autónoma de México. $92 \mathrm{p}$.

35. Treer T, Sprem N, Torcu-Koc H, Sun Y, Piria M. 2008. Length-weight relationships of freshwater fishes of 
Croatia. J Appl Ichthyol 24: 626-628. doi: 10.1111/j.1439-0426.2008.01084.x

36. Vásquez-Torres W. 2004. Principios de nutrición aplicada al cultivo de peces. Villavicencio, Colombia: Universidad de Los Llanos. $66 \mathrm{p}$.

37. Vásquez-Torres W. 2001. Nutrición y alimentación de peces. En: Fundamentos de acuicultura continental. Bogotá, Colombia: Instituto Nacional de Pesca y Acuicultura. p 125-146.

38. Vicentini CA, Franceschini-Vicentini $I B$, Bombonato MTS, Bertolucci B, Lima SG, Santos AS. 2005.
Morphological study of the liver in the teleost Oreochromis niloticus. Inter J Morphol 23: 211-216.

39. Wilson JM, Castro LFC. 2011. Morphological diversity of the gastrointestinal tract in fishes. In: Grossell M, Farrell PA, Brauner JC (eds). The multifuctional gut of fish. USA: Elseiver. p. 2-44.

40. Woynarovich E. 1988. Tambaqui e pirapitinga propagação artificial e criação de alevinos. Brasilia, Brasil: CODEVASF. 68 p. 